\title{
Albert Gier (dir.), Sirenengesänge - Neun Bamberger Vorträge zu Libretto und Musiktheater
}

Héloïse Elisabeth Ducatteau

\section{(2) OpenEdition}

\section{Journals}

Electronic version

URL: https://journals.openedition.org/ifha/11610

DOI: 10.4000/ifha.11610

ISSN: 2198-8943

\section{Publisher}

IFRA - Institut franco-allemand (sciences historiques et sociales)

\section{Electronic reference}

Héloïse Elisabeth Ducatteau, "Albert Gier (dir.), Sirenengesänge - Neun Bamberger Vorträge zu Libretto und Musiktheater", Revue de I'IFHA [Online], Date of review, Online since 16 January 2022, connection on 18 January 2022. URL: http://journals.openedition.org/ifha/11610 ; DOI: https://doi.org/10.4000/ifha. 11610

This text was automatically generated on 18 January 2022.

(C)IFHA 


\section{Albert Gier (dir.), Sirenengesänge - Neun Bamberger Vorträge zu Libretto und Musiktheater}

Héloïse Elisabeth Ducatteau

\section{REFERENCES}

Albert Gier (dir.), Sirenengesänge - Neun Bamberger Vorträge zu Libretto und Musiktheater, Bamberg: University of Bamberg Press, 2018, 264 p, open access. http://dx.doi.org/ 10.20378/irbo-51070 
Un cycle de conférences tenues en 2016 au département de romanistique de l'université de Bamberg est à l'origine de la présente publication. Comme l'introduction le rappelle, la thématique abordée s'enracine depuis la fin des années 80 dans l'institution en question par la création d'un centre de documentation sur la recherche librettistique accompagné de sa revue et suivie de différentes journées d'études. Le périmètre diatopique correspondait au triangle franco-germano-italien. 2016 est l'année de départ à la retraite d'Albert Gier. La même année, les fonds ont été transférés aux archives en recherche musique textuelle de l'université d'Innsbruck. Cette somme de

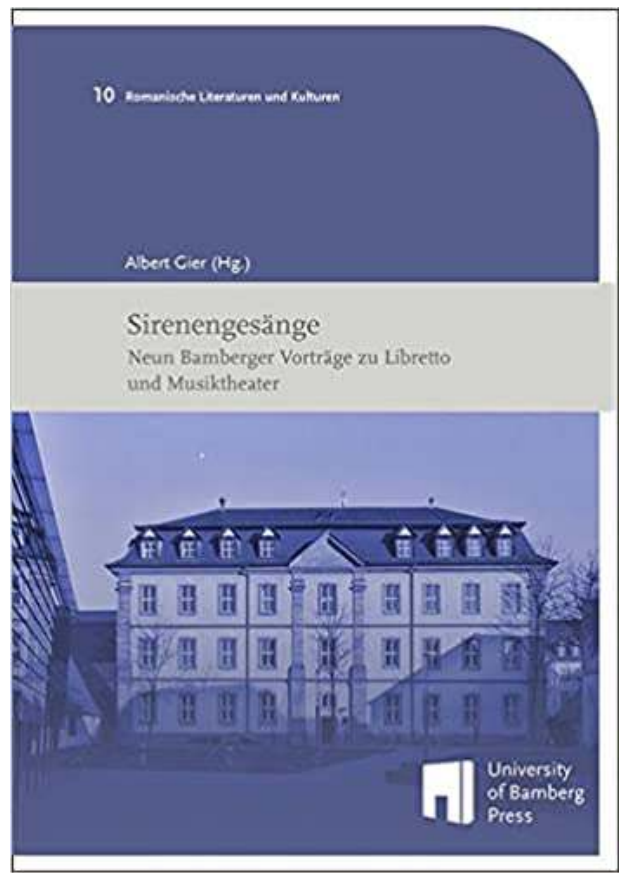
contributions peut donc se lire comme un pot de départ intellectuel concomitant de la fermeture de la chaire en études librettistiques.

Albert Gier ouvre et clôt la succession de contributions. Sa toute première est comparatiste puisqu'il met en regard les libretti en France et en Allemagne. On apprend ainsi qu'en France, cela représentait une activité beaucoup plus lucrative et admirée pour les auteur-es qu'en Allemagne. Les auteurs renommés germaniques se lançaient donc moins dans le libretto. Par ailleurs, le public français était davantage friand de changements de registres et de ruptures de tabous.

Il faut attendre la dernière contribution d'Albert Gier pour que le titre de l'ouvrage soit concrétisé. De façon diachronique, il revient sur L'Odyssée homérienne et un large corpus de libretti. Ceux-ci appartiennent non seulement au répertoire traditionnel avec Rinaldo de Händel et Tannhäuser de Wagner mais aussi à celui plus contemporain avec l'opérette Die Sirene de Leo Fall et l'opéra Sirenen - Bilder des Begehrens und Vernichtens de Rolf Riehms.

Ursula Mathis-Moser qui a participé au déménagement matériel de Bamberg à Innsbruck consacre son article à l'histoire de la recherche en études librettistiques à Bamberg. Puis, elle souligne des différences entre la chanson et l'opéra. Alors que dans le premier genre, le texte est en général créé exprès ou bien il s'agit de la mise en musique d'un texte méconnu, dans le second cas, ce sont souvent des textes littéraires déjà connus du public qui sont réadaptés avant d'être musicalisés.

Adrian La Salvia compare les différentes images de l'Orient véhiculées par le théâtre musical des $\mathrm{XVII}^{\mathrm{e}}$ et $\mathrm{XVIII}{ }^{\mathrm{e}}$ siècles. Il opère une typologie entre Orient merveilleux (décelable dans les épopées chevaleresques de L'Arioste et du Tasse), Orient historique (esclave Roxelane face au tyran Süeyman I ${ }^{\mathrm{er}}$ ) et Orient comique (Serse de Minato).

Richard Armbruster s'attarde quant à lui sur des satires du compositeur Jacques offenbach et de ses productions dans la presse française. Les deux dessinateurs concernés sont STOP (Louis Morel-Retz de son vrai nom) dans le Journal amusant et Albert Robida dans Les contemporains. 
Tina Hartmann se focalise sur l'opéra contemporain, plus particulièrement sur trois œuvres du compositeur Georg Friedrich Haas et du librettiste Händl Klaus. Il soulève la possibilité de l'opéra de s'attaquer à des sujets politiques actuels. Il prend le parti qu'il peut certes ouvrir le débat sur des sujets fondamentaux politiques mais pas sur des sujets brûlants.

Sylvia Tschörner nous plonge dans le travail des traducteurs d'opéras qui sortent actuellement de l'oubli : Il Germanico de Niccolò Coluzzi et Nicola Porpora, Veremonda, l'amazzone in Aragona de Maiolino Bisaccioni et Francesco Cavalli et Le nozze in sogno de Pietro Susini et Pietro Antonio Cesti. Les livrets sont parfois émaillés de mots illisibles ou complètement manquants. Le traducteur doit alors se renseigner intensément sur le contexte pour éviter les incompréhensions.

Frank Piontek plaide pour une revalorisation des opéras de jeunesse de Mozart et du librettiste de la Flûte enchantée, Schikaneder. Selon lui, Lorenzo Da Ponte est peut-être survalorisé ; l'Italien se serait en réalité reposé sur le talent et la notoriété de Mozart.

Norbert Abels se penche sur les opéras d'artistes, c'est-à-dire dans lesquels le personnage principal est un artiste au sens large : peintre, poète ou musicien. C'est l'occasion de revenir sur le topos shakespearien du monde comme théâtre où chacun joue un rôle. De façon macrotemporelle, il va jusqu'en 1979, année de production de Jakob Lenz par Wolfgang Rihm.

\section{INDEX}

Chronological index: Époque contemporaine; Ouvrages transpériodiques Période moderne Subjects: Histoire de la culture ; Histoire de l'art ; Histoire des femmes

\section{AUTHOR}

\section{HÉLOÏSE ELISABETH DUCATTEAU}

Sciences Po Paris (campus de Nancy) 